\title{
Identidad taxonómica de Microlophus heterolepis (Wiegmann, 1835) y Microlophus yanezi (Ortiz, 1980): Dos buenas especies del desierto interior de Chile y Perú
}

\section{Taxonomic identity of Microlophus heterolepis (Wiegmann, 1835) and Microlophus yanezi (Ortiz, 1980): Two good species of the interior desert of Chile and Peru}

\author{
Marcela Vidal ${ }^{1}$, Juan C. Ortiz ${ }^{2 *}$, Gustavo Escobar ${ }^{2} \&$ Fernando Torres-Pérez ${ }^{3}$ \\ ${ }^{1}$ Laboratorio de Ecología y Evolución, Facultad de Ciencias, Universidad del Bío-Bío, Casilla 447, Chillán, Chile. \\ ${ }^{2}$ Departamento de Zoología, Universidad de Concepción, Casilla 160-C, Concepción, Chile. \\ ${ }^{3}$ Instituto de Biología, Pontificia Universidad Católica de Valparaíso, Av. Universidad 330, Campus Curauma, Valparaíso, Chile. \\ *Email: jortiz@udec.cl
}

\begin{abstract}
RESUMEN
En el presente estudio se revisa el estatus taxonómico de Microlophus heterolepis y M. yanezi. A pesar que M. heterolepis es una de las especies nominales de Microlophus en Chile, existen incongruencias evidentes relacionadas con su distribución geográfica, hábitat y ecología, por lo que se intenta entregar una determinación confiable, para lo que se comparó el material tipo con muestras actuales y se revisaron las descripciones originales de ambas especies. Además, se realizaron análisis exhaustivo tanto morfológicos (i.e., escutelación, morfometría lineal y morfometría geométrica) como molecular (cytb). Los resultados muestran que: 1) M. heterolepis fue descrita originalmente para la localidad de Tacna en Perú, 2) las descripciones morfológicas realizadas por Donoso-Barros (1966) corresponden a una lagartija de desierto interior, pero sus características biológicas fueron descritas erróneamente como un habitante del intermareal y 3 ) hay coincidencia con autores previos en que ambas especies son válidas. Sobre la base de estos resultados, se restringe la distribución geográfica de $M$. heterolepis a Perú, mientras que M. yanezi es una especie válidamente descrita para Chile.
\end{abstract}

Palabras clave: Squamata, Tropiduridae, Morfometría geométrica, Análisis Merístico, gen Cyt b.

\begin{abstract}
In the present study, the taxonomic status of Microlophus heterolepis and M. yanezi is reviewed. Although M. heterolepis is one of the identified species present in Chile, incongruity about their geographical distribution, habitat and ecological characteristics are evident, so its true determination is an issue. Type materials and original descriptions of $M$. heterolepis were compared with its closest species M. yanezi, and morphological (scaling, lineal morphometric and geometric morphometric) and molecular analysis (cytb) were performed. The results show that: 1) M. heterolepis was originally described for the locality of Tacna in Peru, 2) morphological descriptions by Donoso-Barros (1966) correspond to a lizard in the desert, but their ecological characteristics were described erroneously as a kind of rocky intertidal, and 3) there is agreement with previous authors that both species are valid. However, based on our results, the geographical distribution of M. heterolepis is restricted to Peru, while M. yanezi is a validly described species from Chile.
\end{abstract}

KeYwords: Squamata, Tropiduridae, Geometric morphometry, Meristic Analysis, Cyt b gene.

\section{INTRODUCCIÓN}

El género Microlophus se distribuye en la vertiente occidental de la cordillera de los Andes, para el cual se han reconocido dos grupos de especies (Etheridge \& de Queiroz, 1988, Frost \& Etheridge, 1989, Frost, 1992) cuya monofilia estaría justificada por caracteres de escamación corporal y pliegues en la piel. El grupo occipitalis se distribuye preferentemente en el norte del Perú y archipiélago de las Galápagos y el grupo peruvianus desde el centro-sur del Perú hasta el norte de Chile (Dixon \& Wright, 1975) y un linaje de las islas Galápagos (Benavides et al. 2007). Además, dentro del grupo peruvianus se ha propuesto la existencia de dos linajes con diferencias distribucionales y ecológicas contrapuestas: uno que habita los sectores rocosos del intermareal del desierto que se alimenta preferentemente de algas y crustáceos y otro que habita en el desierto interior no relacionada directamente al ambiente 
intermareal que se alimenta de insectos y plantas terrestres (Ortíz \& Serey, 1979). Entre las especies reconocidas en el grupo peruvianus de interior se encuentran M. tarapacensis, $M$. theresioides y M. yanezi, mientras que en el grupo de la costa $M$. atacamensis, $M$. heterolepis, M. peruvianus y $M$. quadrivittatus, (Ortiz 1980c).

Históricamente, $M$. heterolepis fue descrita como una especie adscrita al género Tropidurus por Wiegmann (1835) con Tierra típica en Tacna, Perú, pero este autor duda en relacionarla con Lophyrus araucanus descrita por Lesson (1826). Sin embargo, Ortiz 1980b demuestra de $L$. araucanus es sinónima de M. peruvianus del Perú.

Apesar de que Wiegmann (1835) describe a M. heterolepis como una especie típica de desierto interior, Donoso-Barros (1966) entrega antecedentes confusos respecto de la biología de la especie señalando que corresponde a una especie de desierto costero, idea que es continuada por Núñez \& Jaksic (1992) sin mediar un estudio taxonómico que dilucidara las diferencias o semejanzas. Con este nivel de información, Ortiz (1980c) describe una nueva especie con localidad típica en PoconChile en el desierto interior de Arica que vino a incrementar la herpetofauna presente en Chile, pero intensificó la confusión respecto de la procedencia real de M. heterolepis.

Ante la incertidumbre sobre la identidad de estas especies, en este trabajo se revisa el estatus taxonómico de $M$. heterolepis y M. yanezi por medio de análisis morfológicos (escamación, morfometría lineal tradicional y geométrica) y complementado con secuenciación de un fragmento de gen Cytb. Además, se analiza el material disponible en el Museo de Zoología de la Universidad (MZUC) que sirvió de base a Donoso-Barros (1966) y Ortiz (1980c) para proponer estas designaciones.

\section{MATERIALES Y MÉTODOS}

\section{Morfometria LinEAL y anÁlisis Merísticos}

Sustentado en los resultados obtenidos por Ortiz \& Serey (1979) y Ortiz $(1980 \mathrm{~b}, \mathrm{c})$, se evaluaron 13 caracteres morfométricos en 18 individuos adultos de M. yanezi y $M$. heterolepis: (1) longitud de la cabeza (LC); (2) ancho de la cabeza (AnC); (3) alto de la cabeza (AlC); (4) longitud ojo (LO); (5) longitud internarinas (LIN); (6) longitud narinaojo (LNO); (7) longitud narina-punta del hocico (LN-PH); (8) longitud ojo-meato auditivo (LO-MA); (9) longitud del cuello (LCu); (10) longitud hocico-cloaca (LHC); (11) longitud de la extremidad anterior (LEA); (12) longitud de la extremidad posterior (LEP) y (13) longitud del pié (LP). Todas las medidas fueron tomadas con un micrómetro digital Kamasa $( \pm 0.01 \mathrm{~mm})$. Además, se evaluaron 22 caracteres merísticos en 65 individuos adultos de ambas especies siguiendo los caracterres de Ortiz (1980b,c): (1) escamas prenasales (EPren); (2) escamas postnasales (EPostn); (3) escamas supralabiales (ESl); (4) escamas loreales (EL); (5) escamas supraciliares (ESc); (6) escamas supraoculares (ESo); (7) escamas circumorbitales (ECo); (8) escamas postoculares (EPo); (9) escamas temporales (ET); (10) escamas frontoparietales (EFp); (11) escamas frontales (EF); (12) escamas entre las internasales posteriores y las prefrontales (EIp-Pf); (13) escamas nucales a la primera cresta dorsal (EN-PCD); (14) crestas dorsales (CD); (15) escamas infralabiales (EIl); (16) escamas entre la última supralabial y el meato auditivo (EUSl-MA); (17) escamas salientes del meato auditivo (ESMA); (18) escamas gulares (EG); (19) lamelas del cuarto dedo de la mano (LCDM); (20) lamelas del cuarto dedo del pie (LCDP); (21) escamas desde la occipital al inicio de la cola (EO-IC); (22) escamas en la línea media del cuerpo (ELMC). Todas las escamas fueron contabilizadas con una lupa estereoscópica Motic. Los especimenes utilizados para el analisis morfométrico y merístico provienen de la colección herpetológica del Museo de Zoología de la Universidad de Concepción (MZUC).

Previo a los análisis estadísticos de morfometría lineal y merística, los datos fueron examinados para los supuestos de normalidad y homogeneidad de varianza usando las pruebas de Kolmogorov-Smirnov y Levene, respectivamente. Las diferencias en las variables morfológicas fueron probadas por medio de un Análisis Multivariado de Varianza (MANOVA) considerando a la especie como factor (Sokal \& Rohlf, 1995). Las variables morfometricas y meriticas fueron analizadas separadamente usando un Análisis de Componentes Principales (ACP) para determinar si existen grupos morfológicos asociadas a cada especie. Todos los análisis estadísticos se realizaron en el programa Statistica (StatSoft.com, 2016).

AnÁlisis de Morfometria Geométrica

Se examinaron en total 24 individuos adultos de $M$. heterolepis y M. yanezi provenientes de Chile y Perú, que no mostraron deformaciones craneales producto del tiempo de almacenamiento en el Museo. Todo el material está depositado en el Museo de Zoología de la Universidad de Concepción (MZUC). Cada individuo fue fotografiado digitalmente en vista dorsal y lateral de la cabeza con una cámara Canon T4, usando la función supermacro. La calidad de las fotografías fue optimizada con el programa Microsoft Power Point 2010. Se registraron nueve hitos homólogos ("landmarks") localizados en vista dorsal y once en vista lateral de acuerdo con Ibañez et al. (2015), (Fig. 1) que son coincidente con las intersecciones de las escamas del cráneo. La ubicación de los hitos siguió la propuesta de Vidal et al. (2006), pero se agregaron nuevos hitos que son específicos para el género Microlophus. Los hitos fueron digitalizados en TpsDig 2.30 (Rohlf 2016). Para los análisis se siguió los procedimientos de Rohlf \& Slice (1990) and Rohlf et al. (1996). Las coordenadas X, Y de los hitos biológicamente homólogos se alinearon y superpusieron 
usando el método de los mínimos cuadrados, basados en el Análisis Generalizado de Procusto (GPA), que remueve las variaciones no debidas a la forma (Rohlf \& Slice, 1990).

Para obtener la sobreposición y obtener las variables de forma, los individuos alineados se compararon usando TpsRelw versión 1.69 (Rohlf, 2017), que realiza una interpolación "thin-plate spline", proyectando los datos dentro de un plano Euclidiano. De esta manera, las variables de forma se constituyen por componentes uniformes y no uniformes y estas variables se utilizaron posteriormente en un análisis multivariado (Rohlf et al., 1996; Adams \& Rohlf, 2000). Se utilizó TpsRelw versión 1.69 (Rohlf, 2017) para realizar un análisis de componentes principales (ACP, con deformación relativa).
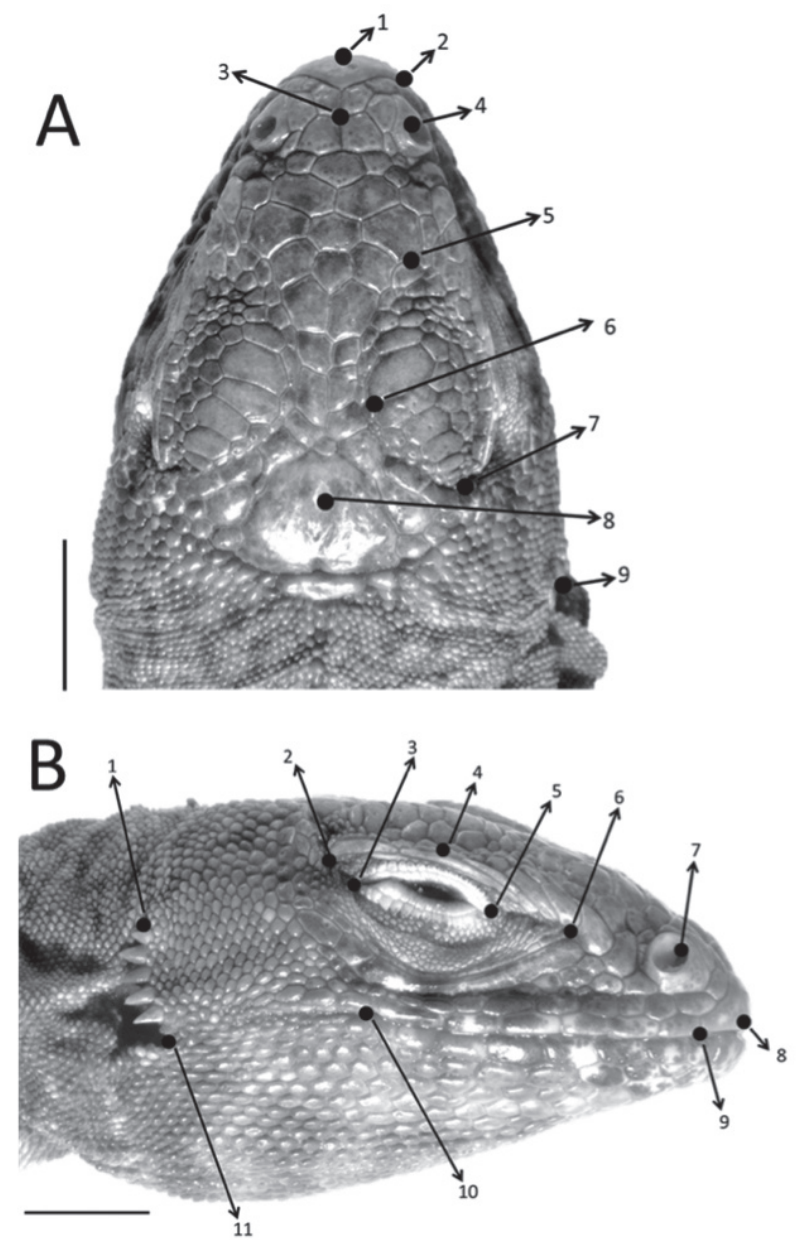

FIGURA 1. Hitos biológicamente homólogos seleccionados para la vista dorsal (A) y vista lateral (B) en las especies de Microlophus consideradas en este estudio. La barra lateral en cada imagen representa $1 \mathrm{~cm}$ en el espécimen real. El esquema está basado en un especimen de Microlophus heterolepis / Biologically homologues landmarks selected for dorsal view (A) and lateral view (B) in Microlophus species considered in this study. The scale in each image represents $1 \mathrm{~cm}$. The scheme is based on a specimen of Microlophus heterolepis.
Las especies se compararon mediante un análisis multivariado de varianza de una vía (MANOVA). Para determinar si la forma contenía un efecto alométrico, se utilizó un análisis de regresión entre el primer eje del ACP (variable de forma) y el tamaño del centroide (CS) (Bookstein, 1991). El tamaño del centroide (CS) fue usado como un índice de tamaño, definido como la suma de las distancias cuadradas de cada hito con el centroide de la forma (Marcus et al. 1996). El tamaño del centroide (Log [CS]) de todos los ejemplares se comparó usando un análisis de varianza de una vía (ANOVA), usando especie como factor.

Análisis Molecular.

El ADN total se aisló de las muestras modificando el método de extracción de sal descrito por Aljanabi \& Martínez (1997). Un fragmento del gen Cyt b de cada ejemplar fue amplificado y secuenciado con dos cebadores nominados como tRNA-Glu y tRNA de acuerdo con Vidal et al. (2016) obtenidos a partir de las secuencias de Iguana iguana (AN: NC002793), Sceloporus occidentalis (AN: NC005960) (LioGluFor: AAACCACCGTTGTTATTCAACTA y LioThrRev: CCATCTTTGGCTTACAAGACCAGAG) (Vidal et al. 2012). Se realizaron diluciones de la muestra de ADN (100 ng/mL) para la amplificación de la reacción en cadena de la polimerasa (PCR). Se amplificó la región del gen Cyt-b por PCR en $25 \mu \mathrm{L}$ de volumen de reacción que constaba $0,3 \mu \mathrm{L}$ Taq (Invitrogen), 2,5 $\mu \mathrm{L}$ PCR Buffer $10 \mathrm{X}, 1,6 \mu \mathrm{L} \mathrm{MgCl}_{2} 50 \mathrm{mM}, 2 \mu \mathrm{L}$ dNTPs $100 \mathrm{mM}, 0,5 \mu \mathrm{L}$ de cada cebador $(10 \mathrm{pmol})$ y $1 \mu \mathrm{L}$ templado de ADN. Las condiciones de amplificación de los ciclos térmicos fueron: desnaturalización inicial a $94{ }^{\circ} \mathrm{C}$ por $5 \mathrm{~min}, 35$ ciclos de desnaturalización de la hebra a $94{ }^{\circ} \mathrm{C}$ durante $30 \mathrm{~s}$, recocido a $53,3{ }^{\circ} \mathrm{C}$ durante 1 min $18 \mathrm{~s}$, extensión del cebador a $72{ }^{\circ} \mathrm{C}$ durante $1 \mathrm{~min} 30 \mathrm{~s}$, y una extensión de $10 \mathrm{~min}$ a $72{ }^{\circ} \mathrm{C}$. El tamaño de los productos de PCR en ambos casos se verificó comparando con una escala de ADN de $100 \mathrm{pb}$ (Invitrogen) en geles de agarosa al 2\%. El ADN amplificado se purificó con el kit QIAGEN de acuerdo con el protocolo del proveedor (Qiagen, USA). Finalmente, todas las muestras se secuenciaron en un secuenciador de ADN automatizado. Las alineaciones de secuencias se editaron con Proseq (Filatov 2002) y se comprobaron visualmente. También se verificaron las secuencias con el programa DnaSP 3.53 (Rozas et al., 2003) para traducirlas en aminoácidos.

Se implementó la aproximación bayesiana para determinar las relaciones filogenéticas de los árboles de genes. En este análisis se utilizaron secuencias no redundantes de M. heterolepis ( $\mathrm{n}=15$ secuencias) y M. yanezi ( $\mathrm{n}=19$ secuencias). Para la reconstrucción filogenética de M. heterolepis-M. yanezi, se eligió una especie como taxon de grupo externo (M. tigris) y sus secuencias se obtuvieron en nuestro laboratorio. El modelo de sustitución de nucleótidos más apropiado $(\mathrm{GTR}+\mathrm{I}+\mathrm{G})$ se estimó con el 
software ModelTest 3.7 (Posada \& Crandall 1998) basado en el Criterio de Información Akaike (AIC). Los árboles filogenéticos fueron reconstruidos con el método Bayesiano de Cadenas de Markov Monte Carlo (MCMC) implementado en el programa BEAST 1.6.2 (Drummond \& Rambaut 2007), el que proporciona un marco flexible para probar hipótesis con datos de secuencia molecular estructurada en el tiempo (Rambaut et al. 2009). Para realizar este análisis se utilizó el reloj relajado no correlacionado (Rambaut et al. 2009, Drummond et al. 2009). El análisis se realizó con 50 millones de generaciones y 1000 generaciones retenidas en un archivo de 10,000 árboles. Las primeras generaciones $(20 \%)$ fueron eliminadas. Se descartaron todos los árboles por debajo del nivel estacionario observado. Estos árboles fueron resumidos en un árbol máximo a posteriori (MAP) con TreeAnotator (parte del paquete BEAST) y visualizados en FigTree v1.2.2.

\section{RESULTADOS}

Morfometría Linear y AnÁlisis Merísticos

Los valores promedio para las variables morfométricas obtenidas para ambas especies se muestran en la Tabla 1. Al considerar el efecto del sexo, no se detectaron diferencias significativas para las especies estudiadas (Lambda de Wilks: 0,008, $\mathrm{p}=0,05$ ) por lo que los datos fueron agrupados en un solo grupo de datos por especie. El análisis multivariado de varianza mostró diferencias significativas para las variables morfológicas (Lambda de Wilks $=0,007$, $\mathrm{p}=0,008$ ) considerando a las especies como factor, donde las variables que mostraron diferencias significativas fueron LC (ANOVA, $\left.\mathrm{F}_{(1,14)}=13,4, \mathrm{p}=0,002\right)$, LCU $\left(\mathrm{F}_{(1,14)}=5,08\right.$, $\mathrm{p}=0,03)$ y $\operatorname{LHC}\left(\mathrm{F}_{(1,14)}=5,36, \mathrm{p}=0,02\right)$. Para estas tres variables, siempre $M$. heterolepis tiende a ser de mayor longitud. En el caso de las variables de escamación (Tabla 2), la variable EFp no mostró varianza por lo que fue eliminada de la matriz de datos. No se registraron diferencias significativas para las características de escamación para todas las variables analizadas (Lambda de Wilks $=0,566$, $\mathrm{p}=0,131)$. Considerando a la especie como factor, no se registraron diferencias significativas al analizar el grupo total de variables cuantificadas (Lambda de Wilks $=0,535, \mathrm{p}$ $=0,073)$, pero solo algunas variables mostraron diferencias significativas entre especies: ET (ANOVA, $\mathrm{F}_{(1,61)}=4,078$, $\mathrm{p}=0,036)$, EII $\left(\mathrm{F}_{(1,61)}=8,623, \mathrm{p}=0,004\right)$, LCDM $\left(\mathrm{F}_{(1,61)}\right.$ $=14,16, \mathrm{p}=0,0003)$ y $\operatorname{LCDP}\left(\mathrm{F}_{(1,61)}=6,85, \mathrm{p}=0,011\right)$, mostrando en todos los casos, que las variables fueron mayores en $M$. yanezi, excepto para la variable LCDP que mostró un valor mayor en $M$. heterolepis (Fig. 2).

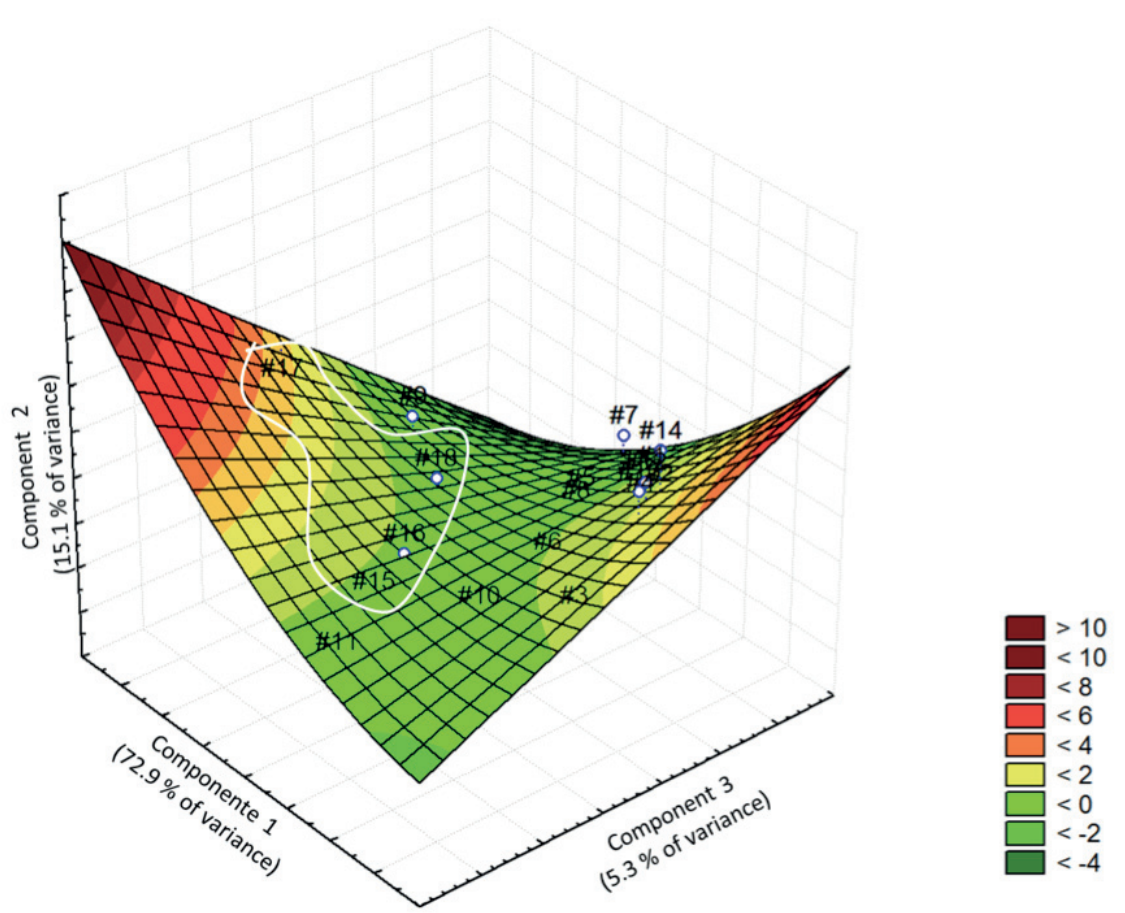

Figura 2. Análisis de Componentes Principales de las variables morfológicas de Microlophus yanezi y Microlophus heterolepis. Los tres primeros componentes acumulan el 93,4 \% del total de la varianza. En la línea blanca se indican los especímenes disponibles de M. heterolepis de la serie tipo / Principal Components Analysis of the morphological variables of Microlophus yanezi and Microlophus heterolepis. The first three components accumulate $93.4 \%$ of the total variance, In the white line the specimens of $M$. heterolepis of the type series are indicated. 
TABLA 1. Caracteres morfológicos (Promedio \pm DE) para machos y hembras de Microlophus heterolepis y Microlophus yanezi (rangos en paréntesis) / Morphological characters (Mean $\pm \mathrm{SD}$ ) for males and females of Microlophus heterolepis and Microlophus yanezi (ranges in parentheses).

\begin{tabular}{|c|c|c|c|}
\hline Caracteres & Abreviatura & $\begin{array}{l}\text { M. heterolepis } \\
(\mathrm{n}=4)\end{array}$ & $\begin{array}{c}\text { M. yanezi } \\
(\mathrm{n}=14)\end{array}$ \\
\hline Longitud de la cabeza & $\mathrm{LC}$ & $\begin{array}{c}22.6 \pm 3.2 \\
(19.1-26.8)\end{array}$ & $\begin{array}{c}20.2 \pm 1.7 \\
(17.8-22.9)\end{array}$ \\
\hline Ancho de la cabeza & $\mathrm{AnC}$ & $\begin{array}{c}16.9 \pm 3.3 \\
(14.6-21.7)\end{array}$ & $\begin{array}{c}14.9 \pm 1.7 \\
(12.5-19.0)\end{array}$ \\
\hline Alto de la cabeza & $\mathrm{AlC}$ & $\begin{array}{c}11.6 \pm 0.7 \\
(10.8-12.4)\end{array}$ & $\begin{array}{c}11.1 \pm 1.0 \\
(9.3-12.6)\end{array}$ \\
\hline Longitud ojo & LO & $\begin{array}{c}6.8 \pm 0.3 \\
(6.5-7.2)\end{array}$ & $\begin{array}{c}6.6 \pm 0.3 \\
(6.2-7.2)\end{array}$ \\
\hline Longitud internarinas & LIN & $\begin{array}{c}3.3 \pm 0.6 \\
(6.5-7.2)\end{array}$ & $\begin{array}{c}3.0 \pm 0.3 \\
(2.8-3.9)\end{array}$ \\
\hline Longitud narina-ojo & LNO & $\begin{array}{c}3.1 \pm 0.4 \\
(3.1-0.4)\end{array}$ & $\begin{array}{c}2.8 \pm 0.2 \\
(2.6-3.5)\end{array}$ \\
\hline Longitud narina-punta del hocico & LN-PH & $\begin{array}{c}2.6 \pm 0.3 \\
(2.4-3.1)\end{array}$ & $\begin{array}{c}2.3 \pm 0.2 \\
(2.6-3.5)\end{array}$ \\
\hline Longitud ojo-meato auditivo & LO-MA & $\begin{array}{c}6.9 \pm 0.9 \\
(6.2-8.0)\end{array}$ & $\begin{array}{c}6.2 \pm 0.7 \\
(5.3-7.9)\end{array}$ \\
\hline Longitud del cuello & $\mathrm{LCu}$ & $\begin{array}{c}13.2 \pm 0.7 \\
(12.6-14.1)\end{array}$ & $\begin{array}{c}12.5 \pm 0.5 \\
(11.3-13.3)\end{array}$ \\
\hline Longitud hocico-cloaca & LHC & $\begin{array}{c}100.9 \pm 13.2 \\
(88.5-118.7)\end{array}$ & $\begin{array}{c}90.6 \pm 7.8 \\
(81.7-109.0)\end{array}$ \\
\hline Longitud de la extremidad anterior & LEA & $\begin{array}{c}73.6 \pm 7.5 \\
(66.3-83.8)\end{array}$ & $\begin{array}{c}67.7 \pm 4.8 \\
(60.9-79.0)\end{array}$ \\
\hline Longitud de la extremidad posterior & LEP & $\begin{array}{c}35.3 \pm 3.5 \\
(31.6-40.0)\end{array}$ & $\begin{array}{c}32.8 \pm 2.1 \\
(30.0-37.6)\end{array}$ \\
\hline Longitud del pié & LP & $\begin{array}{c}41.7 \pm 5.1 \\
(37.6-49.1)\end{array}$ & $\begin{array}{c}38.5 \pm 3.1 \\
(35.2-45.5)\end{array}$ \\
\hline
\end{tabular}

\section{ANÁLISIS DE MORFOMETRÍA GEOMÉTRICA}

Al realizar el análisis multivariado no se registraron diferencias significativas entre las especies para la vista dorsal (Lambda de Wilk $=771 ; \mathrm{p}=0,187$ ) pero sí para la vista lateral (Lambda de Wilk $=0,517 ; p=0,006$ ). Los primeros tres componentes principales explican la mayor parte de la varianza en vista dorsal y lateral (85,7 \% y 56,7 $\%$, respectivamente, Fig. 2 y Fig. 3). En vista dorsal (Fig. 2), el Componente Principal 1 expresa el cambio en la forma de la cabeza sobretodo en la sección media entre las narinas y la abertura timpánica $(63,4 \%$ del total de la varianza de la forma de Procusto), mientras que en vista lateral (Fig. 3 ) expresa el cambio fundamental en la porción anterior a la altura de la sección anterior del ojo $(26,6 \%$ el total de la varianza de la forma de Procusto). En ambos casos, el
Componente principal 1 muestra cambios de alometría $(\mathrm{p}<$ 0,01). El Componente principal 2 en vista dorsal $(14,4 \%$ el total de la varianza de la forma de Procusto) muestra menor variación en la extensión antero-posterior de la cabeza (Fig. 4). Para la vista lateral (19,1\% el total de la varianza), se muestra mayor variación en la extensión antero-posterior del ojo (Fig. 5). Al considerar a ambas especies por separado en el ACP, se observa que M. yanezi muestra un plano curvo cóncavo hacia arriba, mientras que $M$. heterolepis muestra la situación contraria. Lo mismo ocurre para la vista lateral (Fig. 2 y Fig. 3). Los hitos 1, 2 y 4 en vista dorsal son los que muestran la mayor contribución relativa a la variación de la forma que corresponde al hocico y la narina. En vista lateral, hitos 2, 3, 6, 8 y 9 contribuyen mayormente a diferenciar la forma, los cuales están asociados al ojo y el hocico. 


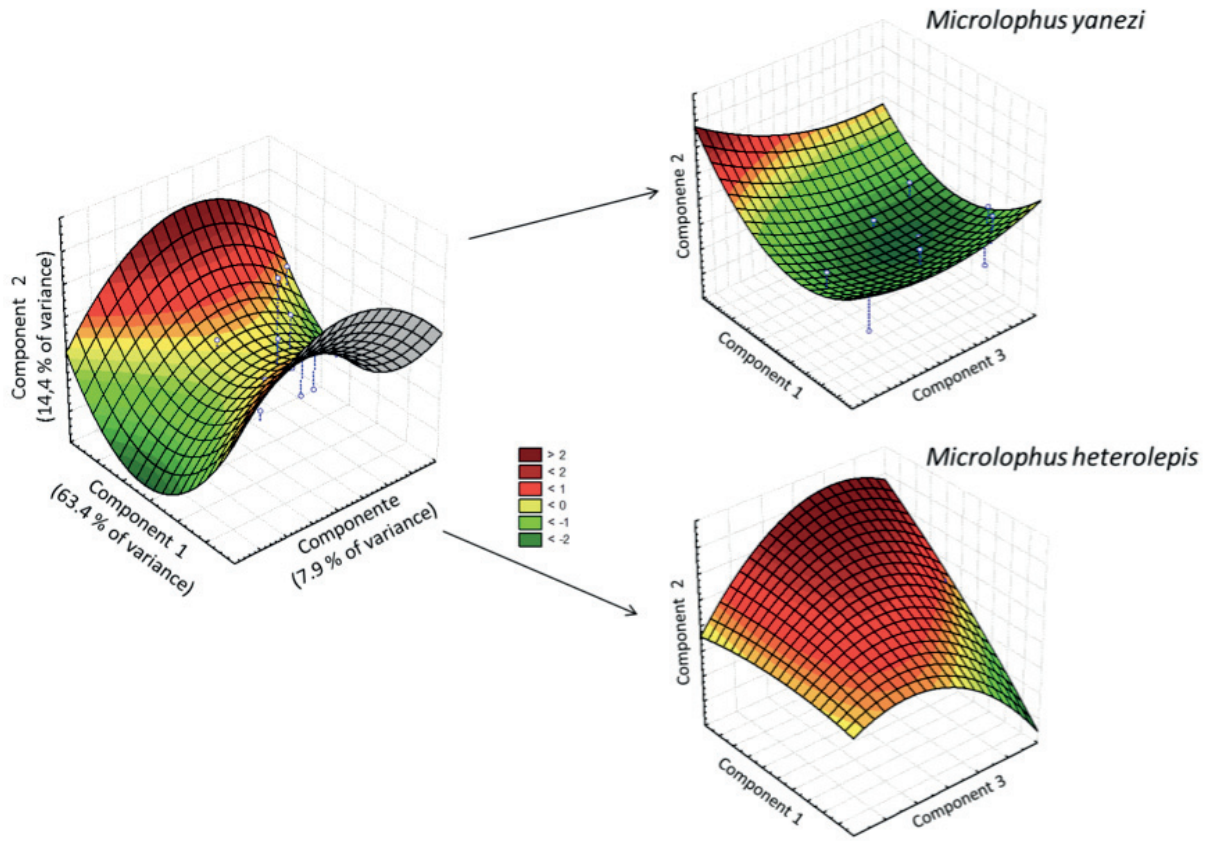

Figura 3. Análisis de Componentes Principales de las variables de forma para la vista dorsal de Microlophus yanezi y Microlophus heterolepis. Los tres primeros componentes acumulan el 85,7 \% del total de la varianza de la forma / Principal Component Analysis of shape variables for the dorsal view of Microlophus yanezi and Microlophus heterolepis. The first three components accumulate $85.7 \%$ of the total variance of the form.

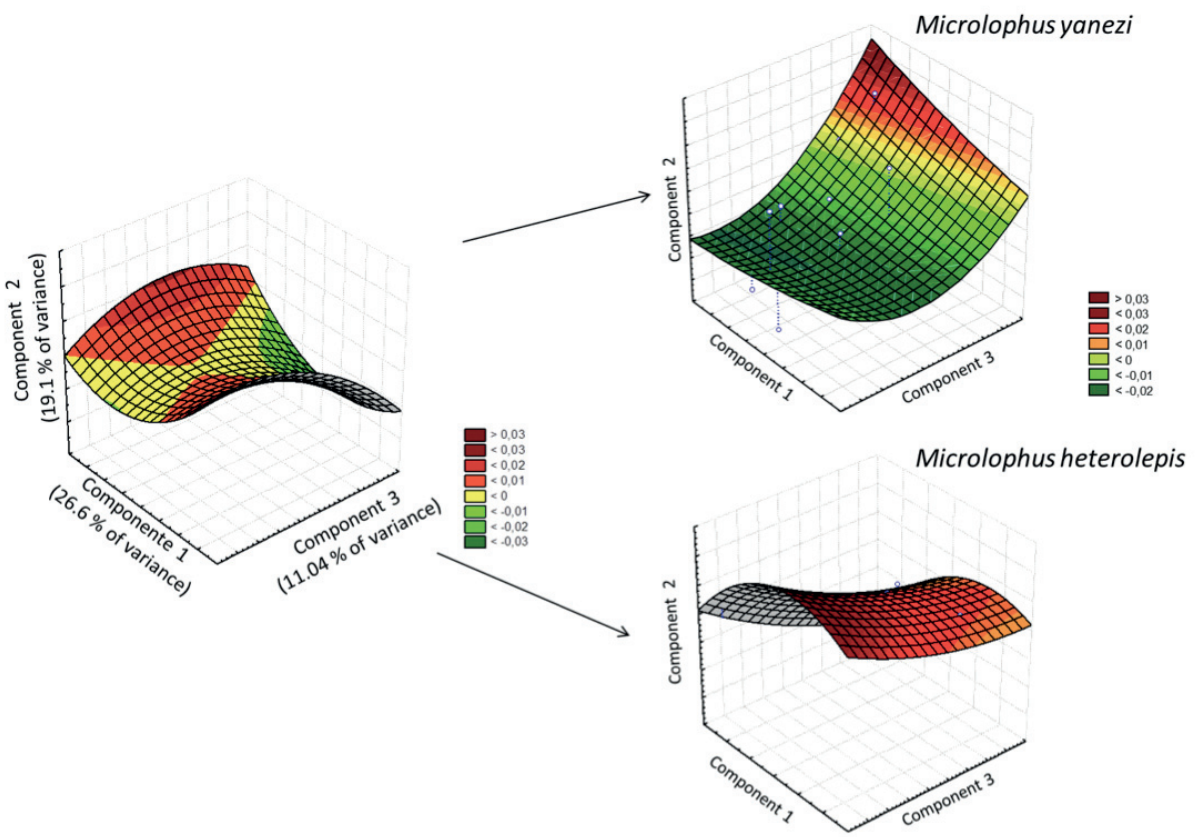

Figura 4. Análisis de Componentes Principales de las variables de forma para la vista lateral de Microlophus yanezi y Microlophus heterolepis. Los tres primeros componentes acumulan el 56,74 \% del total de la varianza de la forma / Analysis of Principal Components of shape variables for lateral view of Microlophus yanezi and Microlophus heterolepis. The first three components accumulate 56.74\% of the total variance of the form. 
Gayana 81(2), 2017

TABLA 2. Caracteres merísticos medidos en Microlophus heterolepis y Microlophus yanezi consideradas en este estudio (rango en paréntesis) / Meristic characters measured in Microlophus heterolepis and Microlophus yanezi considered in this study (ranges in parentheses).

\begin{tabular}{|c|c|c|c|}
\hline Caracteres & Abreviatura & $\begin{array}{c}\text { M. heterolepis } \\
\text { Macho } \\
(\mathrm{n}=6)\end{array}$ & $\begin{array}{c}\text { M. yanezi } \\
\text { Macho } \\
(\mathrm{n}=59)\end{array}$ \\
\hline Escamas prenasales & EPren & $\begin{array}{c}4,0 \pm 0,0 \\
(4-4)\end{array}$ & $\begin{array}{c}3,4 \pm 0,6 \\
(3-5)\end{array}$ \\
\hline Escamas postnasales & EPostn & $\begin{array}{c}4,7 \pm 0,6 \\
(4-5)\end{array}$ & $\begin{array}{c}5,0 \pm 0,5 \\
(4-6)\end{array}$ \\
\hline Escamas supralabiales & ES1 & $\begin{array}{l}6,0 \pm 0,0 \\
(6-6)\end{array}$ & $\begin{array}{c}6,2 \pm 0,6 \\
(5-7)\end{array}$ \\
\hline Escamas loreales & EL & $\begin{array}{c}3,3 \pm 0,6 \\
(3-4)\end{array}$ & $\begin{array}{c}4,1 \pm 0,8 \\
(3-5)\end{array}$ \\
\hline Escamas supraciliares & ESc & $\begin{array}{l}5,0 \pm 0,0 \\
(5-5)\end{array}$ & $\begin{array}{c}5,1 \pm 0,4 \\
(5-6)\end{array}$ \\
\hline Escamas supraoculares & ESo & $\begin{array}{l}6,0 \pm 0,0 \\
(6-6)\end{array}$ & $\begin{array}{c}6,0 \pm 0,0 \\
(6-6)\end{array}$ \\
\hline Escamas circumorbitales & Eco & $\begin{array}{l}15,0 \pm 1,0 \\
(14-16)\end{array}$ & $\begin{array}{l}14,6 \pm 1,5 \\
(12-17)\end{array}$ \\
\hline Escamas postoculares & EPo & $\begin{array}{l}2,0 \pm 0,0 \\
(2-2)\end{array}$ & $\begin{array}{l}2,0 \pm 0,0 \\
(2-2)\end{array}$ \\
\hline Escamas temporales & ET & $\begin{array}{c}7,7 \pm 0,6 \\
(7-8)\end{array}$ & $\begin{array}{c}8,2 \pm 0,6 \\
(7-9)\end{array}$ \\
\hline Escamas frontoparietales & EFp & $\begin{array}{c}2,0 \pm 0,0 \\
(2-2)\end{array}$ & $\begin{array}{c}2,0 \pm 0,0 \\
(2-2)\end{array}$ \\
\hline Escamas frontales & EF & $\begin{array}{c}2,0 \pm 0,0 \\
(2-2)\end{array}$ & $\begin{array}{c}2,5 \pm 0,5 \\
(2-3)\end{array}$ \\
\hline Escamas entre internasales posteriores y prefrontales & EIp-Pf & $\begin{array}{c}3,3 \pm 0,6 \\
(3-4)\end{array}$ & $\begin{array}{c}3,8 \pm 0,4 \\
(3-4)\end{array}$ \\
\hline Escamas nucales hasta primera cresta dorsal & EN-PCD & $\begin{array}{c}4,3 \pm 0,6 \\
(4-5)\end{array}$ & $\begin{array}{c}4,6 \pm 1,0 \\
(3-7)\end{array}$ \\
\hline Crestas dorsales & $\mathrm{CD}$ & $\begin{array}{c}30,3 \pm 2,1 \\
(28-32)\end{array}$ & $\begin{array}{c}27,7 \pm 3,3 \\
(21-34)\end{array}$ \\
\hline Escamas infralabiales & EIl & $\begin{array}{l}7,0 \pm 1,0 \\
(6-8)\end{array}$ & $\begin{array}{c}8,6 \pm 0,5 \\
(8-9)\end{array}$ \\
\hline Escamas entre última supralabial y meato auditivo & EUSl-MA & $\begin{array}{l}12,3 \pm 0,6 \\
(12-13)\end{array}$ & $\begin{array}{l}11,6 \pm 1,0 \\
(10-13)\end{array}$ \\
\hline Escamas salientes del meato auditivo & ESMA & $\begin{array}{l}6,7 \pm 0,6 \\
(6-7)\end{array}$ & $\begin{array}{c}6,0 \pm 0,8 \\
(5-7)\end{array}$ \\
\hline Escamas gulares & EG & $\begin{array}{c}46,7 \pm 1,2 \\
(46-48)\end{array}$ & $\begin{array}{c}46,6 \pm 0,8 \\
(45-48)\end{array}$ \\
\hline Lamelas cuarto dedo de la mano & LCDM & $\begin{array}{c}28,0 \pm 0,0 \\
(28-28)\end{array}$ & $\begin{array}{c}26,6 \pm 0,8 \\
(25-28)\end{array}$ \\
\hline Lamelas cuarto dedo del pie & LCDP & $\begin{array}{c}38,3 \pm 0,6 \\
(38-39)\end{array}$ & $\begin{array}{c}36,4 \pm 1,5 \\
(32-39)\end{array}$ \\
\hline Escamas desde la occipital al inicio de la cola & EO-IC & $\begin{array}{l}123,0 \pm 2,6 \\
(120-125)\end{array}$ & $\begin{array}{l}124,4 \pm 7,9 \\
(110-136)\end{array}$ \\
\hline Escamas en la línea media del cuerpo & ELMC & $\begin{array}{l}156,7 \pm 0,6 \\
(156-157)\end{array}$ & $\begin{array}{l}156,2 \pm 2,8 \\
(145-158)\end{array}$ \\
\hline
\end{tabular}




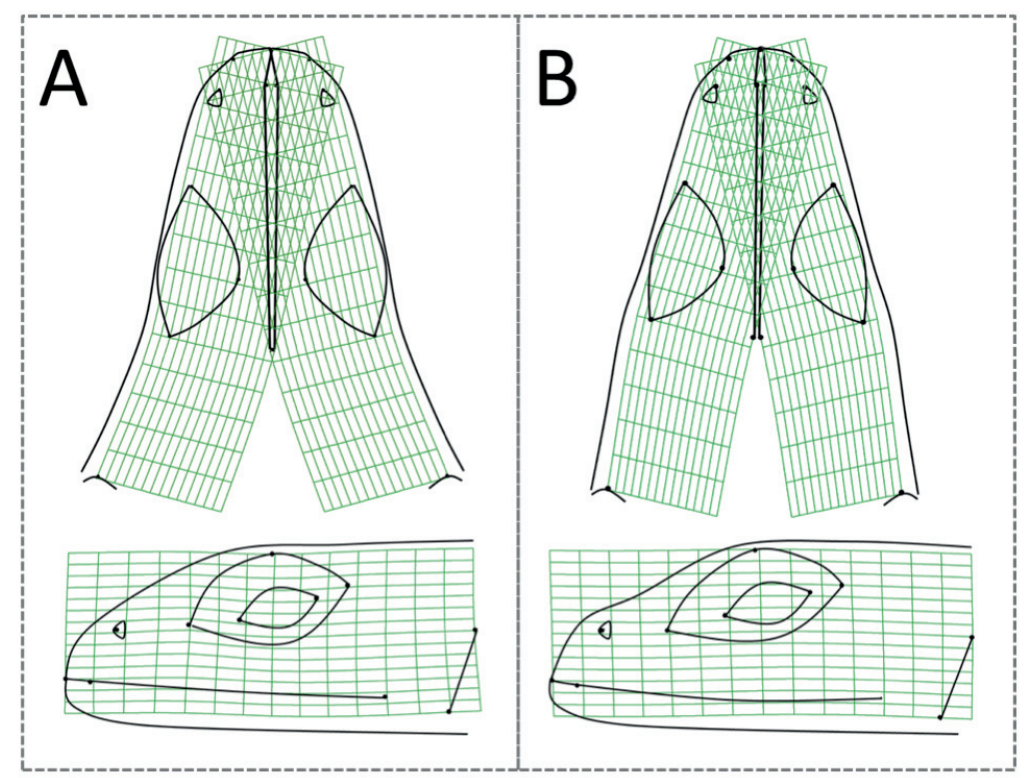

FIGURA 5. Imágenes consenso de las grillas de transformación en vista dorsal y lateral de (A) Microlophus heterolepis y (B) Microlophus yanezi / Images consensus of grids transformation back and side view (A) Microlophus heterolepis and (B) Microlophus yanezi.

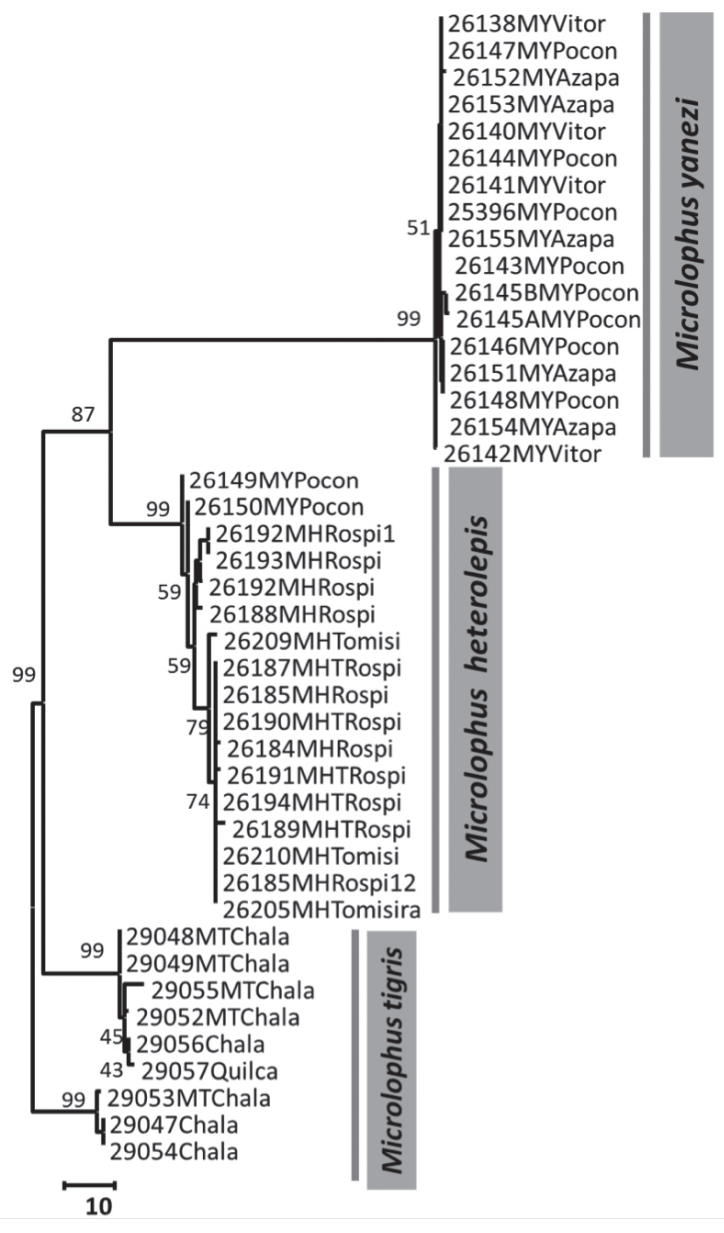

FIGURA 6. Inferencia bayesiana para las especies Microlophus heterolepis y Microlophus yanezi obtenidos desde el gen Citocromo b. El número en las ramas indica el soporte basado en probabilidades del análisis bayesiano, Microlophus tigris fue usado como grupo externo. Los números de especímenes corresponden al número de catálogo del Museo de Zoología de la Universidad de Concepción / Bayesian inference for the species Microlophus heterolepis and Microlophus yanezi obtained from the Cytochrome $\mathrm{b}$ gene. The number in the branches indicates the support based on probabilities of Bayesian analysis, Microlophus tigris was used as an external group. The numbers of specimens correspond to the catalog number of the Museum of Zoology of the University of Concepción. 
Análisis Molecular

Se obtuvieron 43 secuencias de $646 \mathrm{pb}$ del gen Cyt b: 19 individuos de M. yanezi, 15 individuos de M. heterolepis y nueve individuos de $M$. tigris provenientes de siete localidades de Chile y Perú (Tabla 3). Tres grupos son distinguibles a partir del análisis bayesiano (Fig. 6). El primer grupo incluye individuos de M. yanezi provenientes de Caleta Vitor, PoconChile y Valle de Azapa que corresponden a localidades cercanas a la localidad tipo en Chile; el segundo grupo representa las secuencias de individuos provenientes de las localidades de Rospigliosi y Tomisira, las cuales son localidades cercanas a la localidad tipo de $M$. heterolepis en Perú. Sin embargo, los individuos MZUC26149 y 26150 provenientes de la localidad de PoconChile asignables a M. yanezi se asocian al clado de $M$. heterolepis. Finalmente, el último clado corresponde a $M$. tigris que fue utilizado en este estudio como grupo externo, el cual se exhibe como un grupo monofilético distinto de las otras dos especies.

TABLA 3. Ejemplares de donde se obtuvieron las muestras para el análisis molecular. / Individuals from which the samples were obtained for molecular analysis.

\begin{tabular}{|c|c|c|c|}
\hline ESPECIE & MZUC & LOCALIDAD & CoOrdenada \\
\hline Microlophus yanezi & $26138-42$ & Caleta Vitor, Chile & $18^{\circ} 44^{\prime} \mathrm{S}, 70^{\circ} 19^{\prime} \mathrm{O}$ \\
\hline \multirow[t]{2}{*}{ Microlophus yanezi } & 25396 & Pocon Chile, Chile & $18^{\circ} 27^{\prime} \mathrm{S}, 70^{\circ} 03^{\prime} \mathrm{O}$ \\
\hline & $26143-50$ & Pocon Chile, Chile & $18^{\circ} 27^{\prime} \mathrm{S}, 70^{\circ} 03^{\prime} \mathrm{O}$ \\
\hline Microlophus yanezi & $26151-55$ & Valle de Azapa, Chile & $18^{\circ} 29^{\prime} \mathrm{S}, 70^{\circ} 13^{\prime} \mathrm{O}$ \\
\hline \multirow[t]{2}{*}{ Microlophus heterolepis } & $26184-85$ & Rospigliosi, Perú & $18^{\circ} 00^{\prime} \mathrm{S}, 70^{\circ} 16^{\prime} \mathrm{O}$ \\
\hline & $26187-94$ & Rospigliosi, Perú & $18^{\circ} 00^{\prime} \mathrm{S}, 70^{\circ} 16^{\prime} \mathrm{O}$ \\
\hline Microlophus heterolepis & $26205 ; 26209$ & Tomasiri, Perú & $17^{\circ} 50^{\prime} \mathrm{S}, 70^{\circ} 30^{\prime} \mathrm{O}$ \\
\hline \multirow[t]{2}{*}{ Microlophus tigris } & $29047-49$ & 10 km s. de Chala, Perú & $15^{\circ} 52^{\prime} \mathrm{S}, 74^{\circ} 11^{\prime} \mathrm{O}$ \\
\hline & $29052-56$ & 10 km s. de Chala, Perú & $15^{\circ} 52^{\prime} \mathrm{S}, 74^{\circ} 11^{\prime} \mathrm{O}$ \\
\hline Microlophus tigris & 29057 & Quilca, Perú & $16^{\circ} 43^{\prime} \mathrm{S}, 72^{\circ} 25^{\prime} \mathrm{O}$ \\
\hline
\end{tabular}

\section{DISCUSIÓN}

La historia de descripción de estas especies se remonta a los escritos de Wiegmann (1835) quien describe a Tropidurus heterolepis (p. 225, Tab. 17, f. 1) sobre la base de un espécimen infantil con tierra típica Tacna, Perú, que adscribe al género Tropidurus basado en su polidosis típica del género, sin mencionar algún parámetro biológico que la pudiera ubicar ecológicamente en algún ambiente en Chile. En esta publicación, Wiegmann (1835) sugiere una incertidumbre acerca de esta especie al mencionarla como una dudosa sinonimia con Lophyrus araucanus descrita previamente por Lesson (1826) (p. 39-40, Tab. 2, f. 1). Esta última especie, Donoso-Barros (1960) la considera como una subespecie de $M$. peruvianus del litoral de Antofagasta (Chile). Sin embargo, Ortiz (1980a) demuestra que es una especie sinónima de M. peruvianus del Perú. Posteriormente, Ortiz (1980b) al examinar especímenes asociados al litoral de Antofagasta (Chile) consideró que correspondían a otra especie, Microlophus atacamensis. La distribución geográfica de esta especie en la actualidad, está lejana de M. heterolepis por más de $700 \mathrm{~km}$ de distancia y no se relaciona con Lophyrus araucanus que generó la duda en Wiegmann.

Wiegmann (1835) describe a $M$. heterolepis con una coloración típica de una especie de desierto interior, con un tono general café verdoso claro y con la región paravertebral más pálida, sin dibujos pero con manchitas blanquecinas sobre un fondo irregular de color café. Sin embargo, Donoso-Barros (1966) entrega antecedentes confusos respecto de la biología de la especie. De hecho, DonosoBarros (1966, p. 137) señala que M. heterolepis corresponde a "un habitante característico de las rocas de la costa de Arica, sobre las cuales corre y salta con extrema agilidad... por lo general puede observarse a las formas infantiles en las playas arenosas. Los adultos, en cambio, prefieren las rocas. A veces escapa hacia las pozas de agua marina, en las cuales nada ágilmente. Su alimentación está constituida fundamentalmente por productos marinos...". Más aún, Donoso-Barros (1966, p.143) señala sobre M. quadrivittatus que: "esta especie es muy característica en los roqueríos y playas de las cercanías de Iquique...En lo que respecta a sus demás hábitos, puede decirse que son éstos similares a los indicados para heterolepis, propios de las formas 
talasófilas (= de ambiente marino)...". Luego muestra dos ejemplares diferentes en su publicación; en su fotografía de la página LXXI f. 3 a un típico lagarto costero y el dibujo de la página CXII, f. 1 a un típico lagarto de interior. Toda esta descripción fue seguida por numerosos autores, quienes señalaron que $M$. heterolepis correspondía a una especie del intermareal y no al desierto interior (Núñez \& Jaksic 1992) a pesar de que desde la localidad tipo de la descripción original (Tacna, Perú) hasta la costa, hay más de $40 \mathrm{~km}$.

De hecho, con esta incertidumbre, Ortiz (1980c) propuso un análisis taxonómico del género en Chile, señalando que el material aportado por FJF Meyen, que se depositó en el Museo de Berlín, permitió a Wiegmann (1835) describir T. heterolepis con un ejemplar infantil (sintipo ZMB 667) proveniente de Tacna, Perú, Sin embargo, Mertens (1956) señaló la existencia de un segundo ejemplar sintipo (ZMB 666), proveniente de Arica, Chile. Ortiz (1980c) señala que los infantiles que analizó son difíciles de adscribir a una especie definida, por lo que prefiere conservar la especie de Wiegmann y supone un rango geográfico a las cercanías de Arica en Chile debido a la ausencia de barreras entre el desierto interior y la costa. En el desarrollo de su estudio taxonómico de las especies de Tropidurus en Chile, Ortiz (1980c) describe una nueva especie, M. yanezi, con localidad tipo en Poconchile, Valle de Lluta, Chile, distante de la localidad tipo $T$. heterolepis a $53 \mathrm{~km}$, localidad también en el desierto interior. En su trabajo, Ortiz (1980c) la considera diferente de $T$. heterolepis porque la nueva especie tienen menor número de escamas alrededor del cuerpo y menor número de lamelas bajo el cuarto dedo del pie, señalando además, que se diferencia de $T$. peruvianus (hasta ese momento, las subespecies de $T$. peruvianus son eminentemente del intermareal) porque esta nueva especie tiene hábitos que no son costeros.

Los resultados de este estudio indican que, al igual que otros estudios del género (Ortiz 1980 b,c), estas especies no mostraron dimorfismo sexual del tamaño (Ibañez et al 2015). Algunos autores han señalado que los machos de Microlophus crecen hasta que mueren (Watkins, 1997), sin embargo esto no se refleja en un dimorfismo sexual claro en este estudio. Morfológicamente, M. heterolepis mostró ser de mayor tamaño para tres variables corporales, los que pueden considerarse como caracteres diagnósticos para la determinación de ambas especies. Es importante considerar en este punto, que ambas especies son realmente especies de ambiente de desierto interior (Ortiz, 1980c), por lo que diferenciarlas por tamaño es de gran interés. En el caso de las variables de escamación, solo se registraron diferencias para algunas de las variables analizadas (ET, EII, LCDM y LCDP), pero las demás variables no mostraron diferencias a nivel específico. En el pasado, varios autores como DonosoBarros (1966) y Ortiz (1980b,c) utilizaron este tipo de análisis para lograr determinar quiebres en la variabilidad de algunos caracteres con la idea de diferenciar las especies en estudio.
En el caso de las especies de Microlophus presentes en Chile, estas diferencias no fueron claras. Desde la perspectiva de la morfometría geométrica, esta logró diferenciar las especies, pero solo en vista lateral, pero al observar cada uno de los componentes principales que entregan información sobre la forma, se observa que las especies son diferenciables por la conformación de la nube de puntos en el plano. Un problema de este resultado se debe a que el MZUC cuenta solo con cinco especímenes de $M$. heterolepis y a pesar de ello, es interesante encontrar diferencias con este pequeño tamaño de muestra. Por otro lado, se observa que al comparar las especies por separado en el análisis de componente principales, es fácilmente distinguibles que las especies conformar trayectorias evolutivas muy diferentes, esto es, forma cóncava hacia arriba y cóncava hacia abajo. En estos análisis, los hitos que más inciden en diferenciar la forma son el área del ojo y el hocico.

Otro hallazgo importante es la diferenciación genética de las especies analizadas de Chile y Perú, que se relaciona a la distancia geográfica actual de ambas. Con estos resultados, es posible concluir que ambas especies son diferenciables morfológica y molecularmente, por lo que se restringe la distribución geográfica de M. heterolepis al Perú y M. yanezi es considerada una especie válida para el desierto interior de Chile. Futuros estudios que consideren una mayor cantidad de muestras de ambas especies podría clarificar cual es el límite distribucional entre ambas.

\section{AGRADECIMIENTOS}

Este trabajo fue financiado por el Proyecto Fondecyt 1131009 (JCO). Gracias a Juan Pablo Donoso por su ayuda en terreno y a Margarita Ruiz de Gamboa.

\section{REFERENCIAS}

Adams, D., Rohlf, F.J. 2000. Ecological character displacement in Pletodon: biomechanical differences found from a geometric morphometric study. Proceedings of the National Academy of Sciences 97:4106-4111.

Aljanabi, S., Martinez, I. 1997. Universal and rapid salt-extraction of high quality genomic DNA for PCR-based techniques. Nucleic Acids Research 25:4692-4693.

Benavides, E., Baum, R., Mcclellan, D., Sites, J.W. Jr. 2007. Molecular phylogenetics of the lizard genus Microlophus (Squamatai Tropiduridae): Aligning and retrieving indel signal from nuclear introns. Systematics Biology 56(5):776-797.

Bookstein, F.L. 1991. Morphometrics tools for landmark data: geometry and biology. Cambridge University Press, New York.

Dixon, J., Wright, J. 1975. A review of the lizards of the Iguanid genus Tropidurus in Perú. Contributions in Science Natural History Museum, Los Angeles 271:1-39. 
Donoso-Barros, R. 1960. Ecología de los Reptiles Chilenos. Investigaciones Zoológicas Chilenas 6:65-72.

Donoso-Barros, R. 1966. Reptiles de Chile. Universidad de Chile, Santiago, Chile. 458 pp.

Drummond, A.J., Rambaut, A. 2007. BEAST: Bayesian evolutionary analysis by sampling trees. BMC Evolutionary Biology 7:214.

Etheridge, R., De QueIroz, K. 1988. A phylogeny of Iguanidae. In: Estes, R., Pregill, G. (Eds), Phylogenetic relationships of the lizard families: essays commemorating Charles L. Camp, pp. 283-367. Stanford CA: Stanford. Univ. Press.

Filatov, D.A. 2002. ProSeq: A software for preparation and evolutionary analysis of DNA sequence data sets. Molecular Ecology Notes 2:621-624.

Frost, D.R. 1992. Phylogenetic analysis and taxonomy of the Tropidurus group of lizards (Iguania: Tropiduridae). American Museum Novitates 3033:1-68.

Frost, D.R., Etheridge, R. 1989. A phylogenetic analysis and taxonomy of iguanian lizards (Reptilia: Squamata). Museum of Natural History University of Kansas, Miscellaneous publication 81:1-65.

IbÁÑez, S., Vidal, M.A., Ortiz, J.C., Torres-Pérez, F. 2015. Geometric morphometric analysis of the head of Microlophus atacamensis (Tropiduridae) in a latitudinal gradient. Zoological Studies 54:24

Lesson, R.P. 1830. Description de quelques reptiles nouveaux ou peu connus. In: M.L.I. Duperrey, Voyage Autour du Monde Execute par Ordre du Roi, sur la Corvette de La Majeste, La Coquille, exécuté pendant les Années 1822, 1823, 1824 et 1825. 2. Zoologie 2 (1). Arthur Bertrand, Paris: 1-65.

Marcus, L.F., Corti, M., Loy, A., Naylor, G.J.P., Slice, D.E. 1996. Advances in morphometrics. Plenum Press, New York.

Mertens, R. 1956. Studien über die Herpetofauna Peru I. Zur Kenntniss der Iguaniden-Gattung Tropidurus in Peru. Senckenbergiana biologica 37:101-136.

NúÑEZ, H., JAKSIC, F. 1992. Lista comentada de los reptiles terrestres de Chile continental. Boletín del Museo Nacional de Historia Natural, Chile 43:63-91.

Ortiz, J.C. 1980a. Le statut taxinomique de Lophyrus araucanus Lesson, 1831 (Sauria, Iguanidae). Bulletin du Muséum National d'Histoire Naturelle de Paris. 4e. sér. 2 Section A.n²: 599-601.

ORTIZ, J.C. 1980b. Estudios comparativos de algunas poblaciones de Tropidurus de la costa Chilena. Anales del Museo de Historia Natural, Valparaíso 13:267-280.

OrTiz, J.C. 1980c Revisión Taxonómica del Género Tropidurus en Chile. Primera Reunión Iberoamericana de Zoología de Vertebrados, La Rábida. 355-377 pp.

Ortiz, J.C., Serey, I. 1979. Análisis factorial de correspondencias de las especies del género Tropidurus en Chile. Revista de Biología y Medicina Experimentales, Santiago 12:203208.

Posada, D., Crandall, K.A. 1998 Modeltest: testing the model of DNA substitution. Bioinformatics 9:817-818.

Rambaut, A., Ho, S.Y.W., Drummond, A.J., Shapiro, H. 2009. Accommodating the effect of ancient DNA damage on inferences of demographic histories. Molecular Biology and Evolution 26:245-248.

RohlF, F.J. 2016. TPSDIG. Version 2.30 Department of Ecology and Evolution, State University of New York, Stony Brook, NY. URL: http://life.bio.sunysb.edu/morph/.

RoHLF, F.J. 2017. TPSRELW. Version 1.69 Department of Ecology and Evolution, State University of New York, Stony Brook, NY. Website: http://life.bio.sunysb.edu/morph/.

Rohlf, F.J., Slice, D. 1990. Extensions of the Procrustes method for the optimal superimposition of landmarks. Systematic Zoology 39:40-59.

Rohlf, F.J., LoY, A., Corti, M. 1996. Morphometric analysis of old world Talpidae (Mammalia, Insectívora) using partialwarp scores. Systematics Biology 45:344-362.

Rozas, J., Sánchez-del Barrio, J.C., Messeguer, X., Rozas, R. 2003. DnaSP, DNA polymorphism analyses by the coalescent and other methods. Bioinformatics 19:24962497.

SoKal, R., RoHLF, F.J. 1995. Biometry: the principles and practice of Statistics in Biological Research. W.H. Freeman and Co, New York.

Statsoft. 2016. STATISTICA, New Features in STATISTICA 12. URL: http://www.statsoft.com/Products/STATISTICAFeatures/Version-12. Accessed: March 18, 2016.

Vidal, M.A., Veloso, A., Méndez, M. 2006. Insular morphological divergence in the lizard 339 Liolaemus pictus (Liolaemidae). Amphibia-Reptilia 27:103-111.

Vidal, M.A, Ortiz, J.C., Poulin, E., Moreno, P.I., Marín, J.C. 2012. Comparative phylogeography of two codistributed lizards Liolaemus species (Squamata: Tropiduridae) from Southern Chile. Amphibia-Reptilia 33:55-67.

Vidal, M.A., Ibáñez, S., Moreno, P.I., Poulin, E. 2016. Phylogeography of a Patagonian lizard and frog: Congruent signature of southern glacial refuges. Austral Ecology 41(4):399-408.

Watkins, G.G. 1997. Inter-sexual signalling and the functions of female coloration in the tropidurid lizard Microlophus occipitalis. Animal Behaviour 53: 843-852.

Wiegmann, A.F.A. 1835. In: Dr. F.J.F. Meyen: Beiträge zur Zoologie gesammelt auf einer Reise um die Erde. Siebente Abhandlung. Amphibien. Nova Acta Physico-Medica Academia Caesarea Leopoldino-Carolina (Halle) 17:185268.

Recibido: 16.03 .2016

Aceptado: 24.09.2017 